\title{
Discrimination thresholds for haptic perception of volume, surface area, and weight
}

\author{
Mirela Kahrimanovic • Wouter M. Bergmann Tiest • \\ Astrid M. L. Kappers
}

Published online: 26 August 2011

(C) The Author(s) 2011. This article is published with open access at Springerlink.com

\begin{abstract}
The present study investigated the human ability to discriminate the size of 3-D objects by touch. Experiment 1 measured the just noticeable differences (JNDs) for three tasks: (1) discrimination of volume without availability of weight information, (2) discrimination of volume with weight information available, and (3) discrimination of surface area. Stimuli consisted of spheres, cubes, and tetrahedrons. For all shapes, two reference sizes were used (3.5 and $12 \mathrm{~cm}^{3}$ ). No significant effect of task on the discriminability of objects was found, but the effects of shape and size were significant, as well as the interaction between these two factors. Post hoc analysis revealed that for the small reference, the Weber fractions for the tetrahedron were significantly larger than the fractions for the cube and the sphere. In Experiment 2, the JNDs for haptic perception of weight were measured for the same objects as those used in Experiment 1. The shape of objects had no significant effect on the Weber fractions for weight, but the Weber fractions for the small stimuli were larger than the fractions for the large stimuli. Surprisingly, a comparison between the two experiments showed that the Weber fractions for weight were significantly larger than the fractions for volume with availability of weight information. Taken together, the results reveal that volume and weight information are not effectively combined in discrimination tasks. This study provides detailed insight into the accuracy of the haptic system in discriminating objects' size. This substantial set of data satisfies the need for more
\end{abstract}

M. Kahrimanovic $(\triangle) \cdot$ W. M. Bergmann Tiest

A. M. L. Kappers

Helmholtz Institute, Universiteit Utrecht,

Padualaan 8

3584 CH Utrecht, The Netherlands

e-mail: m.kahrimanovic@uu.nl fundamental knowledge on haptic size perception, necessary for a greater understanding of the perception of related properties, as well as of more general perceptual processes.

Keywords Haptics $\cdot$ Size $\cdot$ Touch

\section{Introduction}

Imagine yourself exploring an object with your hands. In order to identify the object, you need to perceive some specific features of that object, such as texture, hardness, shape, and size. In many situations, this process is automatic, fast, and accurate, and probably you would not even think about it. However, from a scientific point of view, it is a fascinating process. It comprises complex perceptual and cognitive mechanisms, and we first need to disentangle each of these mechanisms before trying to understanding in more detail the perceptual system in general. The ability to perceive fundamental object properties (e.g., color and weight) has already been studied since the 19th century, and this topic is still investigated actively. Despite this long history of psychophysical research on object properties, the haptic perception of the size (or volume) of 3-D objects has not been studied in detail so far. However, without sufficient knowledge concerning the perception of objects' size, we will not be able to understand completely the perception of some related properties, such as the weight of objects, since it has been shown that size has a systematic influence on other percepts (e.g., the size-weight illusion). Also, more detailed knowledge of size perception would aid in the understanding of processes like object recognition and the control of manual actions (e.g., grasping), which 
may be relevant for interactions with objects in virtual settings, such as teleoperation. Therefore, the present study investigated the human ability to discriminate the size of 3-D objects.

When discriminating by touch the size of two small same-shaped objects, we can grasp each object between two fingers and make a judgment of the distance between the two fingers, which corresponds to the length of the object. A number of studies have been performed on the just noticeable differences (JNDs) of length for objects held between two fingers. The stimuli used in these studies were either calipers or blocks varying in length. The JNDs were often reported in proportion to the reference stimulus, resulting in a Weber fraction. The reported Weber fractions for length discrimination varied highly, ranging from .02 to .11 (Dietze, 1961; Durlach et al., 1989; Evans \& Howarth, 1966; Kelvin, 1954; Langfeld, 1917; Stevens \& Stone, 1959). These studies provided some insight into the human ability to discriminate haptically the length of objects. However, size discrimination of 3-D objects comprises more than merely the discrimination of length. Instead of exploring the object between two fingers, we could also take the object in the whole hand and explore it by enclosure. This will provide information about properties such as the global shape, the volume, and the total surface area of the object, properties that would not be available to the haptic sense when an object is only grasped between two fingers. In this situation, the size of a 3-D object might be best described by its volume. Lederman and Klatzky (1987) demonstrated that enclosure was the stereotypical exploratory procedure that was used by participants when they were asked to judge the volume of a 3-D object. Although, a detailed investigation of the JNDs for volume is required for a comprehensive understanding of haptic size perception of 3-D objects, there are no studies on the accuracy of the volume judgment by way of enclosure. In addition to the scientific importance, knowledge concerning volume perception may also be applied in the field of remote handling and teleoperation.

The present study investigated haptic perception of volume and focused on the following four questions:

1. Effect of shape. Previous studies on haptic volume perception were based mainly on the influence of the shape of objects on perceived volume (Kahrimanovic, Bergmann Tiest \& Kappers, 2010; Krishna, 2006). They revealed that the volume judgment is strongly biased by the objects' shape. For example, tetrahedrons were perceived as larger than cubes and spheres, and cubes were perceived as larger than spheres (Kahrimanovic et al., 2010). Because of the strong influence of shape on the volume percept, a study on the JND for volume should investigate whether volume discrimination thresholds also differ between shapes. Therefore, the present study investigated the JNDs for volume for three different shapes (i.e., tetrahedrons, cubes, and spheres), which were the same as those in the previous experiment on perceptual biases. This created the possibility of comparing the effect of shape on volume perception of differently shaped objects with the effect of shape on volume perception of same-shaped objects.

2. Effect of size. Previous studies on the discrimination of the length of objects showed that the JND for length increased with reference length. However, these JNDs were not a constant proportion of the original stimulus value, indicating that length perception is not in accordance with Weber's law. The studies showed that in particular, the Weber fractions for the small stimuli were relatively large (Dietze, 1961; Durlach et al., 1989; Gaydos, 1958; Stevens \& Stone, 1959). The present study tested whether comparable patterns would occur for volume discrimination. Preferably, a study like this should include a large range of reference stimuli. The choice of the stimulus range that could be used for an experiment with unimanual enclosure of objects is, however, limited by the size of the hand. Therefore, for the present stimuli and exploration procedure, it is not possible to test Weber's law comprehensively. By a comparison of the thresholds for two substantially different reference sizes, the present experiment will at least shed some light on the effect of size on the JNDs for volume. On the basis of the studies on length discrimination, we hypothesized that the fraction for the small reference stimulus would be larger than the one for the large reference stimulus.

3. Volume versus surface area. The Kahrimanovic et al. (2010) study showed that the haptic volume judgments were biased by the objects' shape because the judgments were based on the surface area of objects, instead of the volume itself. Hence, during the volume judgment task, an object with a larger surface area was perceived as being larger in volume, as compared with a differently shaped object with the same physical volume but a smaller physical surface area. This suggests that the haptic system does not perceive the volume of an object directly but extracts it from other object properties. We suggested that the judgment might be based on the property that was most salient during exploration, which was the surface area in this case. The same study showed also that when participants were asked to discriminate two differently shaped objects according to surface area, their performance did not differ from their performance during the volume task. This indicates that the task (i.e., compare 
objects according to volume or surface area) did not influence the judgment and that the same strategies were used for both volume and surface area judgments. Related to these findings, the present study investigated whether these tasks would have an influence on JNDs when same-shaped objects were compared. It mightbe hypothesized that for both the volume and the surface area tasks, the same strategies would be used. Therefore, no effect of task on discrimination thresholds would be expected.

4. Influence of availability of weight information. In addition to the use of the volume or the surface area to distinguish the size of objects, the weight may also provide relevant information. From experience, we know that a heavier object should be larger than a lighter object made of the same material. We tested whether availability of weight information, which correlated perfectly with volume information in the present study, would influence the volume judgment. It has been shown that the biases for volume perception are smaller in conditions with weight information than in those without, indicating that weight information is used appropriately to aid volume judgment (Kahrimanovic et al., 2010). Therefore, it might be hypothesized that volume discrimination of equally shaped objects would also be aided by additional weight cues.

\section{Experiment 1}

\section{Method}

Participants A group of 8 students (3 of them male, 5 female) from Utrecht University participated in the first experiment. Their mean age was 23 years $(S D=2$ years), and they were all right-handed as tested by Coren's handedness questionnaire (Coren, 1993). All were naïve as to the purpose of the experiment, and they provided written informed consent. They were paid for their participation.

Stimuli Two sets of tetrahedrons, cubes, and spheres made out of brass were used as stimuli. Both sets consisted of stimuli that could fit in one hand, with volumes ranging from 2 to $5 \mathrm{~cm}^{3}$ and from 10 to $14 \mathrm{~cm}^{3}$. For each object shape, two references were used: a small reference of $3.5 \mathrm{~cm}^{3}$ for the first range and a larger one of $12 \mathrm{~cm}^{3}$ for the second range. Set 1 consisted of completely solid objects (see Fig. 1), with the mass of these objects covarying consistently with their volume. This set was used for the task with weight information. Set 2 was identical to the first
A

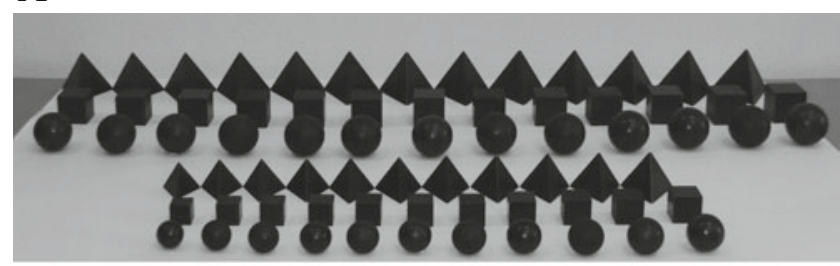

B

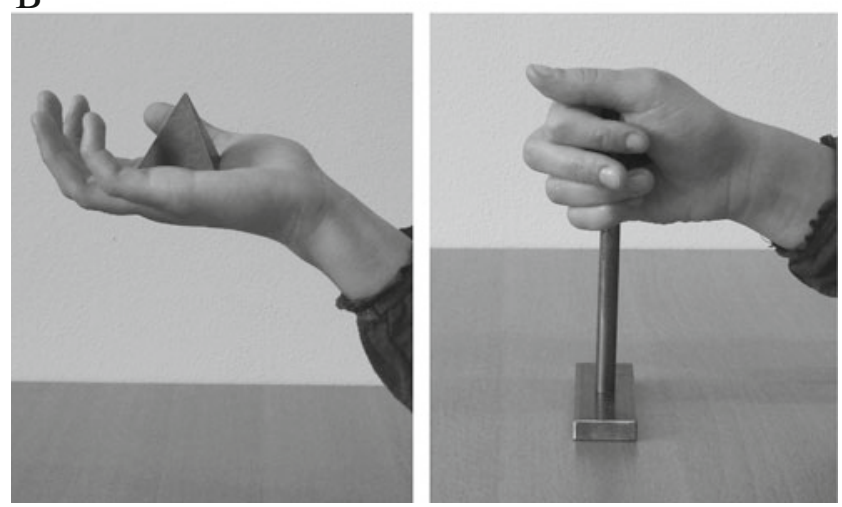

Fig. 1 a The two ranges of objects from the stimulus set with the completely solid objects (set 1 ). The objects from stimulus set 2 were identical to those but included a small cylindrical hole, creating the possibility of placing them on stands. b A participant holding the largest tetrahedron free in the hand (left) and exploring the same stimulus on the stand (right)

one, with the exception that in each object, a small cylindrical hole (diameter of $1 \mathrm{~mm}$ ) was made to be able to place the objects on stands and, thereby, eliminate the availability of weight information. ${ }^{1}$

Conditions A 3 (shape) $\times 2$ (size) $\times 3$ (task) within-subjects design was used, resulting in 18 different conditions. The three different shapes were tetrahedrons, cubes, and spheres. The size factor was determined by the size $(3.5$ and $12 \mathrm{~cm}^{3}$ ) of the reference stimulus. The task factor consisted of three different tasks. Either the participants were asked to explore pairs of objects on stands (conditions without weight information) and to compare them according to either their volume or surface area, or they were asked to explore pairs of objects that were placed successively in their hands (condition with weight information) and to compare them according to volume. The 18 conditions were performed within nine sessions. Shape and task were randomized between sessions, and the two different sizes were randomized within a session. Furthermore, the order of the test and reference stimuli within each trial was randomized. Each session lasted for about $1 \mathrm{~h}$,

\footnotetext{
${ }^{1}$ Stimulus set 2 was not used in the conditions with weight information. Due to the small cylindrical hole in each of the objects, the weight of these objects did not correspond precisely with their volumes.
} 
resulting in about $9 \mathrm{~h}$ per participant for the complete experiment.

Procedure After receiving the instructions, the participants were blindfolded. For the conditions without mass information, the stands (12 cm high) on which the objects were placed were fixed on the table in front of the participant, with a center-to-center distance between the stands of $10 \mathrm{~cm}$. The participants were familiarized, with some practice trials, with the location of the stands and the objects on them. They were asked to start each trial from a fixed reference point, such that they could locate the stimuli easily in just one smooth arm movement. To start a trial, the experimenter indicated that the participant could explore the first stimulus, which was always the stimulus on the right side. After the palm of the hand had touched one side of the stimulus, the stimulus had to be enclosed to perceive the shape and the volume as thoroughly as possible. The participants were instructed to enclose the stimulus maximally. After the initial enclosure, they were allowed to open their hand and to enclose the stimulus in another way. They were not allowed to use only the fingers for the exploration process, since this would reduce the judgment to a judgment of the length. After exploration of the first stimulus, the participant moved his/her hand toward the second stimulus and explored it in the same way. The participant had to indicate which of the two stimuli was either larger in volume or larger in surface area, depending on the condition.

For the conditions with weight information, each participant was asked to rest the elbow of his/her right arm on the table and to hold the hand in a horizontal position with the palm facing upward. The experimenter placed the stimuli, one after the other, in the center of the palm. The participant was asked to enclose each stimulus, similar to the exploration of the stimuli on stands, in order to perceive its shape and volume. After exploration of the two stimuli, the participant had to indicate which of the two had a larger volume. In these conditions, the participant could also perceive the weight of the stimuli. However, no explicit information was given concerning the weight of the objects or the possibility of using weight information during the volume judgment.

Data collection The data were collected with a method of constant stimuli that consisted of two parts. The first part was the same for all participants and was used for a rough estimation of the discrimination threshold. This part consisted of a reference stimulus and test stimuli that differed in steps of $0.5 \mathrm{~cm}^{3}$ from the reference stimulus, up to the outer stimuli of the range. Each combination of test and reference stimuli in this part was tested 4 times. The stimulus range in the second part was based on the performance within this first set. If performance was worse than a specified criterion, ${ }^{2}$ the same set as that in the first part was used. Each combination of reference and test stimuli was repeated 6 more times in order to end up with ten repetitions for each combination. If performance was better than this criterion (see Fig. 2), a narrower set of test stimuli, differing in steps of $0.25 \mathrm{~cm}^{3}$, was used, and each stimulus combination was presented 10 times in total. The data from both parts were taken together for the analyses.

Data analyses For each combination of reference and test stimuli in the different conditions, the fraction was calculated with which the participant selected the test stimulus to be the larger in volume/surface area. A weighted cumulative Gaussian distribution $(f)$ as a function of the volume of objects $(V)$ was fitted to the data with the maximum-likelihood procedure, using the following equation:

$f(V)=\frac{1}{2}\left\lfloor 1+\operatorname{erf}\left(\frac{V-V_{r e f}}{\sigma \sqrt{2}}\right)\right\rfloor$,

where the parameter $\sigma$ is a measure of the $84 \%$ discrimination threshold and the parameter $V_{\text {ref }}$ corresponds to the volume of the reference stimulus that was used. The measured discrimination threshold indicates the sensitivity of participants in perceiving differences between two objects (for an example, see Fig. 2). Note that the volume of objects was used as a parameter in all conditions and also when the participants were asked to compare the objects in terms of surface area. In this way, the values of the discrimination thresholds were not influenced by the use of different parameters in different conditions. Each discrimination threshold was then divided by the volume of the reference stimulus, resulting in the Weber fraction.

\section{Results}

Figure 3 shows the average Weber fractions for the two reference sizes, the three tasks, and the three shapes. As can be seen in the figure, the average Weber fractions ranged from .11 for the large sphere in the surface area task to .20 for the small tetrahedron in the volume task with weight information. A 3 (task) $\times 3$ (shape) $\times 2$ (size) repeated measures ANOVA performed on the Weber fractions revealed no significant effect of the factor task, $F(2,14)=1.9, p=.19$, but the main effects of size and

\footnotetext{
${ }^{2}$ The criterion was determined with a computer simulation. For different specified discrimination thresholds and different criteria, we used a Monte Carlo simulation to generate 100 data sets based on a binomial distribution and calculated for each data set the discrimination threshold. The difference between the specified and the calculated discrimination thresholds proved to be smallest at a criterion of about $83 \%$ correct responses, indicating that the discrimination threshold would be determined most accurately with that criterion level.
} 


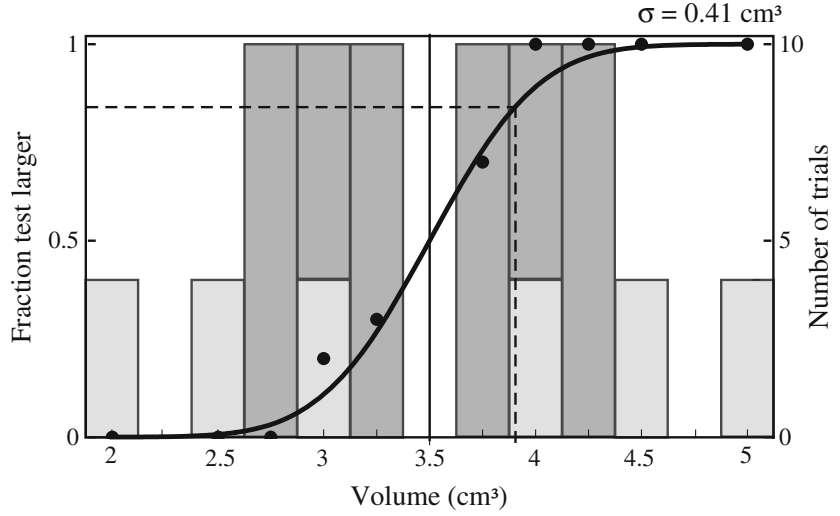

Fig. 2 An example of data collected for a small reference, for which the the second part used a narrower range. The bar chart shows the number of repetitions for each test volume (right scale). The light-gray bars indicate the trials from the first part of the data collection procedure. The dark-gray bars indicate the trials from the second part. The curve shows the fitted function through the measured data points (left scale). The vertical line at $3.5 \mathrm{~cm}^{3}$ indicates the volume of the reference stimulus. The dashed line specifies the place of the $84 \%$ threshold. The value of this discrimination threshold, $\sigma$, is shown in the right top corner of the figure. If the range in the first and second parts was kept the same, the data points would be only at the positions indicated by the light-gray bars, and all those points would be measured 10 times

shape were significant, $F(1,7)=12, p<.01$, and $F(2,14)=$ $11, p<.001$, respectively. Furthermore, there was also a significant interaction effect between shape and size, $F$ $(2,14)=7.4, p<.005$. No other interactions were significant $(p>.05)$. The significant effects will be explored further in the following section.

Effects of shape and size The main effect of size revealed that the Weber fractions for the smaller stimuli, on average $.16(S E=.01)$, were significantly larger than the Weber fractions for the larger stimuli, on average $.13(S E=.01)$. The significant main effect of shape indicated that the Weber fractions differed depending on the shapes that were compared. The average fractions for the tetrahedron, cube, and sphere were $.17(S E=.01), .15(S E=.01)$, and .127 $(S E=.007)$, respectively. In order to explore further the significant interaction effect between shape and size, a repeated measures ANOVA with shape as the withinsubjects factor was performed for each reference size. No significant effect of shape on the Weber fractions was found for the large reference, $F(2,14)=2.6, p=.11$. On the other hand, for the small reference, the effect of shape was highly significant, $F(2,14)=16, p<.001$. Bonferroni corrected pairwise comparisons showed that, for the smaller reference, the Weber fraction for the tetrahedron $(.19, S E=.01)$ was significantly larger than both the fraction for the sphere $(.133, S E=.007)$ and the fraction for the cube $(.16, S E=.01)$, with $p<.005$ and .05 , respectively, for the two comparisons. The comparison
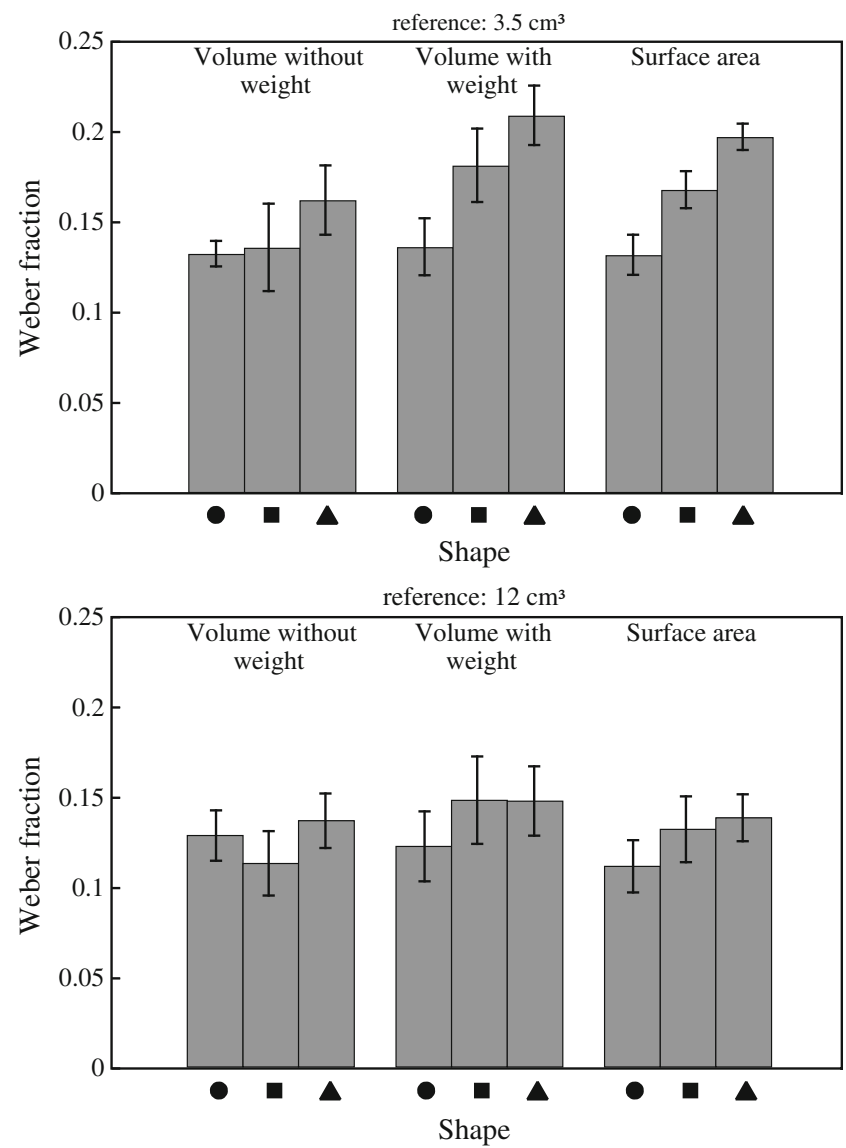

Fig. 3 The average Weber fractions for the different conditions, with standard errors of the means indicated by the error bars. The volume of the objects was used as a parameter for the calculation of the fractions in all conditions. The 2-D symbols stand for their 3-D counterparts

between the Weber fractions of the cube and the sphere failed to reach significance $(p=.18)$.

Furthermore, paired sample $t$-tests were performed to compare the fractions for the small and the large objects for all three shapes individually. These tests showed that the fractions for the small objects were significantly larger than those for the large objects for the cube and the tetrahedron, $t(7)=2.8, p<.05$, and $t(7)=4.2, p<.005$, respectively. For the sphere, the two fractions did not differ significantly, $t(7)=1.4, p=.21$.

\section{Experiment 2: Discrimination thresholds for weight}

Experiment 1 revealed no differences between the thresholds in conditions with and without weight information. This finding is interesting since we expected the performance to be better when more relevant information was available. In order to explore this finding further, a second experiment was performed in which the participants were 
asked to discriminate the weight of objects. Discrimination thresholds for weight have already been investigated extensively (for a short overview, see Jones, 1986). However, the reported thresholds varied widely (Weber fractions ranged from .03 to .13), and they were measured with rather different stimuli and exploration strategies than those used in the present study. It would not be very informative to compare the results from the first experiment with those thresholds from the literature. Therefore, the second experiment was necessary for a better understanding of the processes involved in haptic size perception with availability of weight information.

Method

Design The design was the same as that for the volume condition with weight information in the previous experiment. The only difference was that the participants in this second experiment were asked to discriminate the objects according to their weight instead of their volume. No information was provided about the relationship between the volume and the weight of the objects. However, the participants were instructed explicitly that it was important, for the purpose of the experiment, to enclose the objects at each trial. The three different shape conditions were performed in different sessions, and the size factor was randomized within a session. Each session lasted for about $1 \mathrm{~h}$, resulting in about $3 \mathrm{~h}$ per participant for the complete experiment.

Participants A group of 8 participants (5 of them male, 3 female; mean age, 22 years) took part in this experiment. They were all right-handed as tested by Coren's test (Coren, 1993). They provided informed written consent and were paid for their participation. No participants in this experiment had taken part in Experiment 1.

\section{Results}

The Weber fractions for weight are shown in Fig. 4, together with the relevant Weber fractions for volume from
Experiment 1. The ANOVA performed on these weight fractions revealed no significant effect of shape, $F(2,14=$ $2.1, p=.16$, but the effect of reference size was significant, $F(2,7=37, p<.001$. The average Weber fraction for weight for the small reference $(.35, S E=.04)$ was significantly larger than the fraction for the large reference $(.18, S E=.03)$. Furthermore, a significant interaction effect between shape and size was found, $F(2,14)=3.9, p<.05$. However, post hoc analyses revealed that the effect of shape was not significant for the large reference, $F(2,14)=0.059$, $p=.94$, or for the small reference, $F(2,14=3.7, p=.051$. The significant interaction effect can be related to a trend toward a smaller Weber fraction for the cube than for the other two objects in the condition with the smaller reference.

Comparison of the Weber fractions for weight and those for volume revealed that the Weber fraction for weight was significantly larger than the one for volume for the small sphere and tetrahedron and for the large sphere $(p<.05$, two-tailed $t$-test).

\section{General discussion}

The present study investigated the ability to discriminate the size of same-shaped small 3-D objects during unimanual haptic exploration. The effects of shape, size, and three different tasks on the discrimination threshold were investigated in Experiment 1. Experiment 2 investigated the discrimination thresholds for weight. The following sections discuss the observed effects.

\section{The effect of shape}

The results from Experiment 1 showed that the size discriminability of 3-D objects was significantly influenced by their shape for the small reference. The Weber fraction for the tetrahedron was significantly larger than the fractions for the cube and the sphere. A possible explanation for this effect of shape may be related to the saliency of specific object properties. The discrimination of objects may be disrupted by the presence of the edges and,
Fig. 4 Average Weber fractions for weight (Experiment 2) and volume (Experiment 1) for the three different shapes and two different sizes. The 2-D symbols stand for their 3-D counterparts. The error bars represent the standard errors of the means
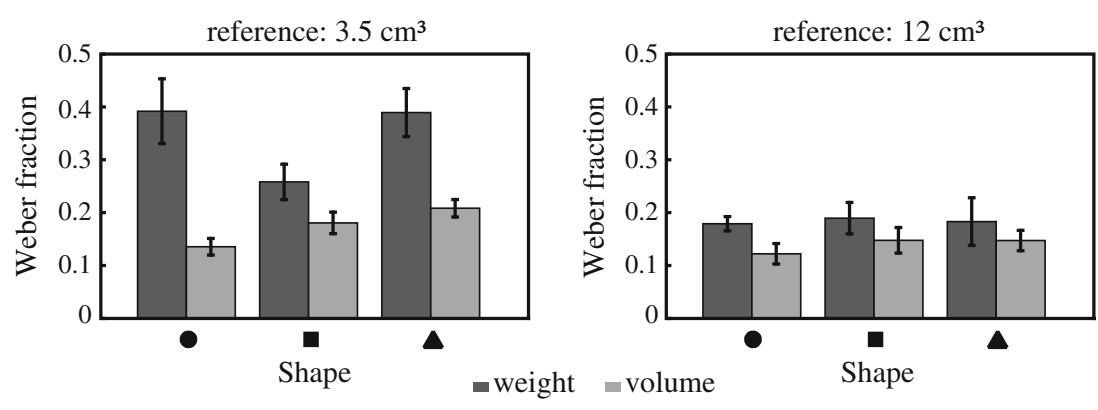
especially, the sharp vertices of the tetrahedrons, which were probably rather salient during exploration. The cube also included edges and vertices, but these were probably less salient. These suggestions can be related to studies on the saliency of object features and studies on the interference of edges with haptic shape perception. Plaisier, Bergmann Tiest and Kappers (2009) showed that edges and vertices were the salient features that aided the haptic search for a target object between distractors. They showed that finding a tetrahedron between spheres is easier than finding a cube between spheres, as manifested in steeper search slopes for the cube-sphere search. Panday, Bergmann Tiest and Kappers (2011) showed that presence of the salient edges disrupted the participants' ability to perceive haptically the orientation of blocks. In conditions with edges, the discrimination threshold was increased by $50 \%$, as compared with conditions in which the exploration was restricted in such a way that participants were not allowed to touch the edges. The present results suggest that the presence of salient edges also disrupts size perception of small objects.

\section{The effect of size}

The present results showed that the Weber fractions for cubes and tetrahedrons, but not for spheres, were larger for the small reference size. This effect may be related to the way in which the objects were apprehended, by enclosure in the hand: Small finger movements might provide more information for the large than for the small reference size. Similarly, Klatzky, Lederman and Reed (1987) suggested that the low discriminability and salience of size during haptic exploration might be related to the exploratory procedure of enclosure, which provides mainly gross information. This, however, does not account for the lack of the effect of size on discriminability of spheres. The present effect of size on the threshold also resembles the findings of studies on size discrimination of objects held between two fingers, which showed that the absolute discrimination thresholds for size increased with an increase of reference size but that size perception is not in accordance with Weber's law (Dietze, 1961; Durlach et al., 1989; Evans \& Howarth, 1966; Stevens \& Stone, 1959).

Volume versus surface area

Discrimination thresholds of volume and surface area were the same. Furthermore, the effect of shape on the threshold was the same for the two tasks. This suggests that the same strategy was used for the discrimination of volume and of surface area. Our previous study on perceptual biases (Kahrimanovic et al., 2010) showed that for the comparison of differently shaped objects, participants used comparable strategies for both volume and surface area judgments. The present data suggest a similar effect when same-shaped objects are compared. Another similarity between discrimination of same-shaped and differently shaped objects becomes apparent when the values of the Weber fractions from the two studies are compared. The previous study revealed an average Weber fraction of $.16(S E=.02)$, which is comparable to the fractions obtained in the present experiment. The use of surface area may be related to its salience during enclosure. We propose that the haptic system does not perceive volume directly but that volume judgment is based on "simpler" dimensions that are salient during the exploration.

\section{Effect of weight information}

Experiment 1 also investigated the influence of the availability of weight information on the volume judgment. The results showed that the Weber fractions for volume in conditions with and without weight were the same. This finding is surprising, since we expected that weight information would aid the volume judgment. Kahrimanovic et al. (2010) showed that the addition of weight information to the volume judgment of differently shaped objects resulted in less bias. It is also the case that cue combination theories predict that integration of two cues will result in a better performance than is obtained with a single cue. At least in our study, however, in which the participants were not informed explicitly about the relation between volume and weight, two cues were no better than one.

\section{Thresholds for weight discrimination}

Experiment 2 of the present study investigated the influence of shape and reference size on the discrimination of the weight of 3-D objects. The results showed that shape did not influence the Weber fractions for weight, which were significantly larger for the small reference stimulus. The fractions - on average, .29-are larger than those reported in other studies, which ranged between .03 and .13 (Jones, 1986). This difference may be related to the way the objects were explored: In the present study, the object was enclosed; in previous studies, it was hefted in the palm or lifted by the fingers. Furthermore, Weber fractions for weight were larger than those for volume with weight information; this was mainly true for the small objects. We observed that, when asked to judge weight, but not when asked to judge volume, the majority of participants performed hefting movements after enclosing the object. This suggests that participants did not attend to weight when asked to judge volume. This is not surprising, since weight discrimination (at least in our study) was more difficult. 


\section{Conclusions}

The present study revealed that unimanual haptic discrimination of the size of 3-D objects is influenced by their shape and size. Furthermore, volume discrimination was not aided by the availability of weight information or vice versa, and the discrimination of volume did not differ from the discrimination of surface area. In addition, the present study showed that the shape of 3-D objects had no significant influence on haptic perception of the weight of these objects but that weight discrimination was influenced by the size of the objects. Finally, comparison of Weber fractions for volume and weight revealed that the fractions for weight were larger, indicating that discrimination of the weight of objects is a more difficult task than discrimination of their volume.

Acknowledgments This research was supported by a grant from The Netherlands Organization for Scientific Research (NWO).

Open Access This article is distributed under the terms of the Creative Commons Attribution Noncommercial License which permits any noncommercial use, distribution, and reproduction in any medium, provided the original author(s) and source are credited.

\section{References}

Coren, S. (1993). The left-hander syndrome: The causes and consequences of left-handedness. New York: Vintage.

Dietze, A. G. (1961). Kinaesthetic discrimination: The difference limen for finger span. Journal of Psychology, 51, 165-168.
Durlach, N. I., Delhorne, L. A., Wong, A., Ko, W. Y., Rabinowitz, W. M., \& Hollerbach, J. (1989). Manual discrimination and identification of length by the finger-span method. Perception \& Psychophysics, 46, 29-38.

Evans, G. B., \& Howarth, E. (1966). The effect of grip-tension on tactile-kinaesthetic judgement of width. Quarterly Journal of Experimental Psychology, 18, 275-277.

Gaydos, H. F. (1958). Sensitivity in the judgment of size by fingerspan. The American Journal of Psychology, 71, 557-562.

Jones, L. A. (1986). Perception of force and weight: Theory and research. Psychological Bulletin, 100, 29-42.

Kahrimanovic, M., Bergmann Tiest, W. M., \& Kappers, A. M. L. (2010). Haptic perception of volume and surface area of 3-D objects. Attention, Perception, \& Psychophysics, 72, 517-527.

Kelvin, R. P. (1954). Discrimination of size by sight and touch. Quarterly Journal of Experimental Psychology, 6, 23-34.

Klatzky, R. L., Lederman, S., \& Reed, C. (1987). There's more to touch than meets the eye: The salience of object attributes for haptics with and without vision. Journal of Experimental Psychology. General, 116, 356-369.

Krishna, A. (2006). Interaction of senses: The effect of vision versus touch on the elongation bias. Journal of Consumer Research, 32, $557-566$.

Langfeld, H. S. (1917). The differential spatial limen for finger span. Journal of Experimental Psychology, 2, 416-430.

Lederman, S. J., \& Klatzky, R. L. (1987). Hand movements: A window into haptic object recognition. Cognitive Psychology, 19, 342-368.

Panday, V., Bergmann Tiest, W. M., \& Kappers, A. M. L. (2011, June). The influence of edges as salient features in haptic shape perception of 3D objects. In Proceedings of the IEEE World Haptics Conference (pp. 529-532). Istanbul.

Plaisier, M. A., Bergmann Tiest, W. M., \& Kappers, A. M. (2009). Salient features in 3-D haptic shape perception. Attention, Perception, \& Psychophysics, 71, 421-430.

Stevens, S. S., \& Stone, G. (1959). Finger span: Ratio scale, category scale, and JND scale. Journal of Experimental Psychology, 57, 91-95. 